\title{
Photon Spectra Measured above Operating Floor of Unit 3 Reactor at Fukushima Daiichi Nuclear Power Station
}

\author{
Yoshihiko TANIMURA ${ }^{1, *, \dagger}$, Hideo HIRAYAMA ${ }^{1,2}$, Kenjiro KONDO ${ }^{1,2}$, \\ Hiroshi NAGATA ${ }^{1}$, Kouhei IWANAGA ${ }^{1}$ and Seishirou SUZUKI ${ }^{1}$ \\ ${ }^{1}$ Secretariat of Nuclear Regulation Authority, 1-9-9 Roppongi, Minato-ku, Tokyo 106-8450, Japan \\ ${ }^{2}$ High Energy Accelerator Research Organization, 1-1 Oho, Tsukuba-shi, Ibaraki 305-0801, Japan
}

\begin{abstract}
Photon energy spectra were measured above the operating floor of unit 3 reactor at the Fukushima Daiichi Nuclear Power Station by using a CdZnTe semiconductor spectrometer. The spectrometer was installed in a lead collimator to measure the photons from the area directly below the detector. The collimator and spectrometer were lifted up by a huge crane and set above the operating floor. The photon spectra were derived by unfolding the pulse height spectra measured using the spectrometer. The response function of the spectrometer was calculated with the MCNP-4C code and was used as an input parameter of the unfolding code MAXED. It was found from the photon energy spectra that low-energy photons with energy below $0.4 \mathrm{MeV}$ were dominant above the operating floor. These spectra are fundamental data for evaluating the dose reduction effect by setting up shields on the operating floor.
\end{abstract}

KEYWORDS: photon energy spectrum, CdZnTe spectrometer, unfolding, Fukushima Daiichi Nuclear Power Station, unit 3 reactor, operating floor, ${ }^{137} \mathrm{Cs},{ }^{134} \mathrm{Cs}$

\section{Introduction}

The Unit 3 reactor at Fukushima Daiichi Nuclear Power Station was severely damaged by hydrogen explosions due to an accident attributed to the Great East Japan Earthquake that

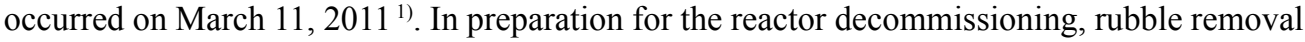
and decontamination have progressed at the operating floor on the fifth floor of the Unit 3 reactor (hereinafter referred to as "operating floor") and the removal of fuel from the spent fuel pool has been scheduled. For fuel removal, several tasks have been planned to be conducted remotely. However, some tasks need to be conducted through manned operations, such as the installation of the basement girder. Since the operating floor continues to produce a high dose rate due to the high-density contamination caused at the time of the explosion, dose reduction through decontamination measures have been implemented, such as peeling the floor surface on the assumption of surface contamination, and removing the rubble. However, sufficient

\footnotetext{
* Corresponding author, E-mail: tanimura.yoshihiko@jaea.go.jp

$\dagger$ Present, Japan Atomic Energy Agency

DOI : 10.15669 /fukushimainsights. Vol.4.495

(C) 2021 Atomic Energy Society of Japan. All rights reserved.

Originally published in Transactions of the Atomic Energy Society of Japan (ISSN 1347-2879), Vol. 15, No. 3, p.129-132

(2016) in Japanese. (Japanese version accepted: May 25, 2016)
} 
reduction has not yet been achieved. Therefore, the installation of a shield for further dose reduction is planned ${ }^{2)}$.

The Secretariat of the Nuclear Regulation Authority assumed that high-density contamination might be observed at places other than the surfaces, such as the lower surface of the shield plug located on top of the reactor, which could be a radiation source. If a place other than the surfaces is contaminated, the contribution of scattered rays with low energy will be increased by penetration through the concrete, etc. Therefore, we attempted to measure the energy spectra of the photon beams emitted upwards from the operating floor surface in order to confirm the assumption described above. The spectra thus obtained not only enable the determination of the exposure dose of workers in more detail, but also could be the basic data required to optimize the thickness and material of the shield to be installed.

\section{Measuring Method}

We used a CdZnTe semiconductor detector with a crystal size of $1 \mathrm{~cm} \times 1 \mathrm{~cm} \times 1 \mathrm{~cm}(\mathrm{GR}-1$ manufactured by Kromek) ${ }^{3)}$ for the measurement of photon beam energy spectra. By installing a CdZnTe semiconductor in a lead collimator (with a diameter of $0.8 \mathrm{~cm}$ ), we eliminated the photon beams entering from the periphery and measured only the photon beams emitted upwards from the operating floor surface. Figure 1 shows the layout of the collimator and detector.

The collimator was equipped with a tablet PC for data acquisition and mobile Wi-Fi router to measure the spectra, while monitoring the status remotely. The collimator and the detector were lifted with a 600 -t crane and moved to the measurement position on the operating floor of the Unit 3 reactor so that the sensitive volume of the CdZnTe detector was located at a height of approximately $50 \mathrm{~cm}$ above the operating floor surface. As typical samples of locations on the operating floor, 24 positions were selected, mainly on the shield plug on top of the reactor, where the dose rate is considered to be particularly high and a major radiation source, as shown in Figure 2. We conducted measurements at these 24 positions on November 19, 2015.

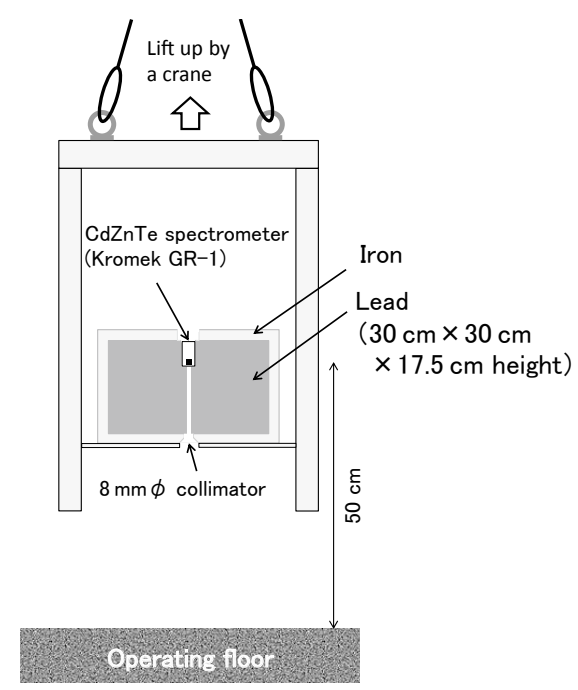

Figure 1 Schematic drawing of the lead collimator and the CdZnTe semiconductor spectrometer 

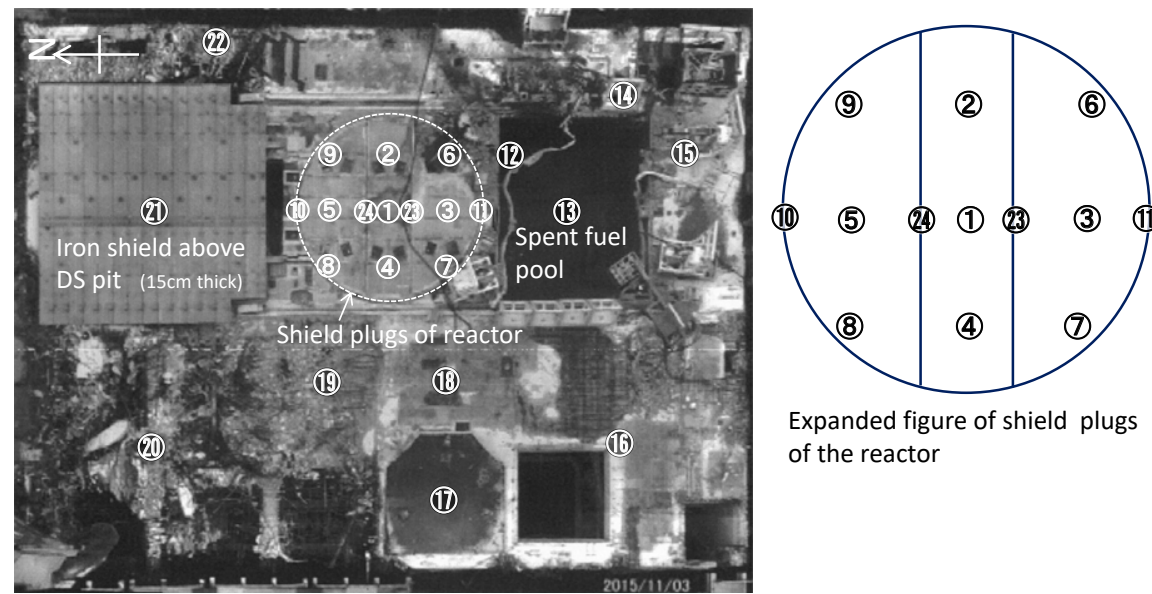

Expanded figure of shield plugs of the reactor

Figure 2 Measurement points of photon spectra above the operating floor of unit 3 reactor

\section{Measurement Results}

Figure 3 shows the pulse height distribution of the CdZnTe semiconductor detector measured at a height of $50 \mathrm{~cm}$ above the reactor shield plug, spent fuel pool and the iron shield plate on the DS pit. At the joint of the plug (Example: 24), etc.), the photoelectric peaks formed by the $0.662 \mathrm{MeV} \gamma$-rays from ${ }^{137} \mathrm{Cs}$ and the $0.605 \mathrm{MeV}$ and $0.8 \mathrm{MeV} \gamma$-rays from ${ }^{134} \mathrm{Cs}$ were observed. On the other hand, no photoelectric peak formed by $\gamma$-rays from radioactive Cs was observed on the spent fuel pool (13) and iron shield plate on the DS pit (21)), etc. In addition, at all measurement positions, no photoelectric peak formed by $\gamma$-rays from any radioactive nuclide, other than ${ }^{137} \mathrm{Cs}$ and ${ }^{134} \mathrm{Cs}$, was observed. From these results, it is estimated that the radiation dose on the operating floor is attributable to ${ }^{137} \mathrm{Cs}$ and ${ }^{134} \mathrm{Cs}$.

\section{Derivation of Photon Energy Spectra}

The photon energy spectra were derived by unfolding the pulse height distribution measured by the CdZnTe detector. The response of the detector, which was used in the unfolding procedure, was calculated as follows. First, a calculation model was created by simulating the structures of the CdZnTe semiconductor detector and collimator as closely as possible. Then, the response of the detector to the photons that passed through the collimator was calculated using the Monte Carlo code MCNP-4C ${ }^{4}$ ) and the model. Subsequently, the energy resolution of the $\mathrm{CdZnTe}$ semiconductor detector was evaluated using the pulse height distribution relative to the $\gamma$-rays from a ${ }^{137} \mathrm{Cs}$ radiation source in order to broaden the calculated response using the Gaussian distribution, which could reproduce the measured pulse height distribution ${ }^{\mathrm{a}}$. The response function of the $\mathrm{CdZnTe}$ semiconductor detector was created by changing the energy of incident photons by up to $1.5 \mathrm{MeV}$ at $10-\mathrm{keV}$ increments to calculate the pulse height distribution relative to the photon beams of a total of 150 energies.

\footnotetext{
${ }^{a}$ Compared to the photons entering from the front face of the detector (on the collimator side), the photons entering from the rear face (top) of the detector tend to cause lower resolution due to the scattering substances such as the electrical circuit. In this report, we created the response function assuming the photons entered from the front face.
} 


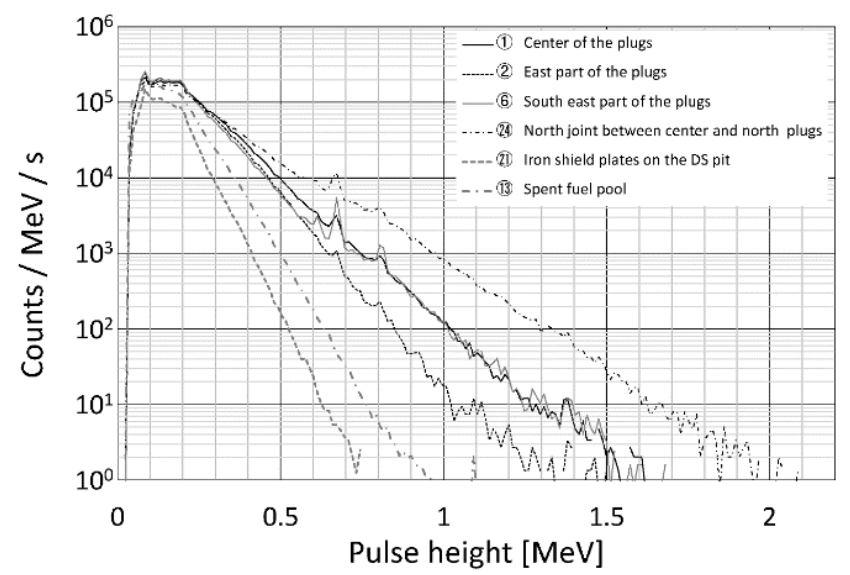

Figure 3 Pulse height spectra measured above the operating floor of unit 3 reactor

For unfolding the pulse height distribution, we used MAXED code, which was developed by PTB (Physikalisch-Technische Bundesanstalt, Germany) and included in the UMG-3.3 code package. MAXED code is based on the principle of the maximum entropy method and can estimate the photon energy spectrum, thus reproducing the measured pulse height distribution by using the above response function ${ }^{5}$. For the 23 positions excluding measurement position (11), where the influence of the pile-up could not be ignored due to the high counting rate, we derived the energy spectra of the photon beams that were emitted upwards from the operating floor surface as shown in Figure 4.

The vertical axis shows the fluence rate per unit area of the photons entering the CdZnTe semiconductor detector through the lead collimator with a diameter of $0.8 \mathrm{~cm}$.

\section{Discussion}

From the photon energy spectra shown in Figure 4, the contribution of the scattered rays with decreased energy of $0.4 \mathrm{MeV}$ or lower was large and the influence of the direct radiations from ${ }^{137} \mathrm{Cs}$ and ${ }^{134} \mathrm{Cs}$ was low at all positions. This result suggests that the contamination source is not only on the operating floor surface, but also in deeper areas, such as the lower part of the shield plug ${ }^{6}$. The dose reduction effect of the iron shield placed on the operating floor surface increases as the photon beam energy decreases. Therefore, when the thickness of the shield is determined based on the assumption of direct radiation with high energy, the dose reduction effect of the iron shield may be greater than expected in an environment where the contribution of scattered rays is large, as described above.

As shown in Figure 4, peaks were observed in the scattered rays at approximately $0.07 \mathrm{MeV}$ and $0.2 \mathrm{MeV}$, on and around the shield plug. The peak at $0.07 \mathrm{MeV}$ is caused by the characteristic X-rays of lead generated in the collimator. Table 1 shows the relation between the scattering angle based on Compton scattering and the energy of the scattered rays. As shown in Figure 1, since no shield plate for the CdZnTe semiconductor detector was placed on top of the collimator, this structure enabled photons scattering downwards in the air in the upper portion of the collimator to enter the detector. Among the scattering angles shown in Table 1, the angle of approximately $180^{\circ}$ corresponds to the position directly above the collimator, and the energy of the scattered rays is $0.18-0.2 \mathrm{MeV}$ in this case. Since this energy of the scattered 


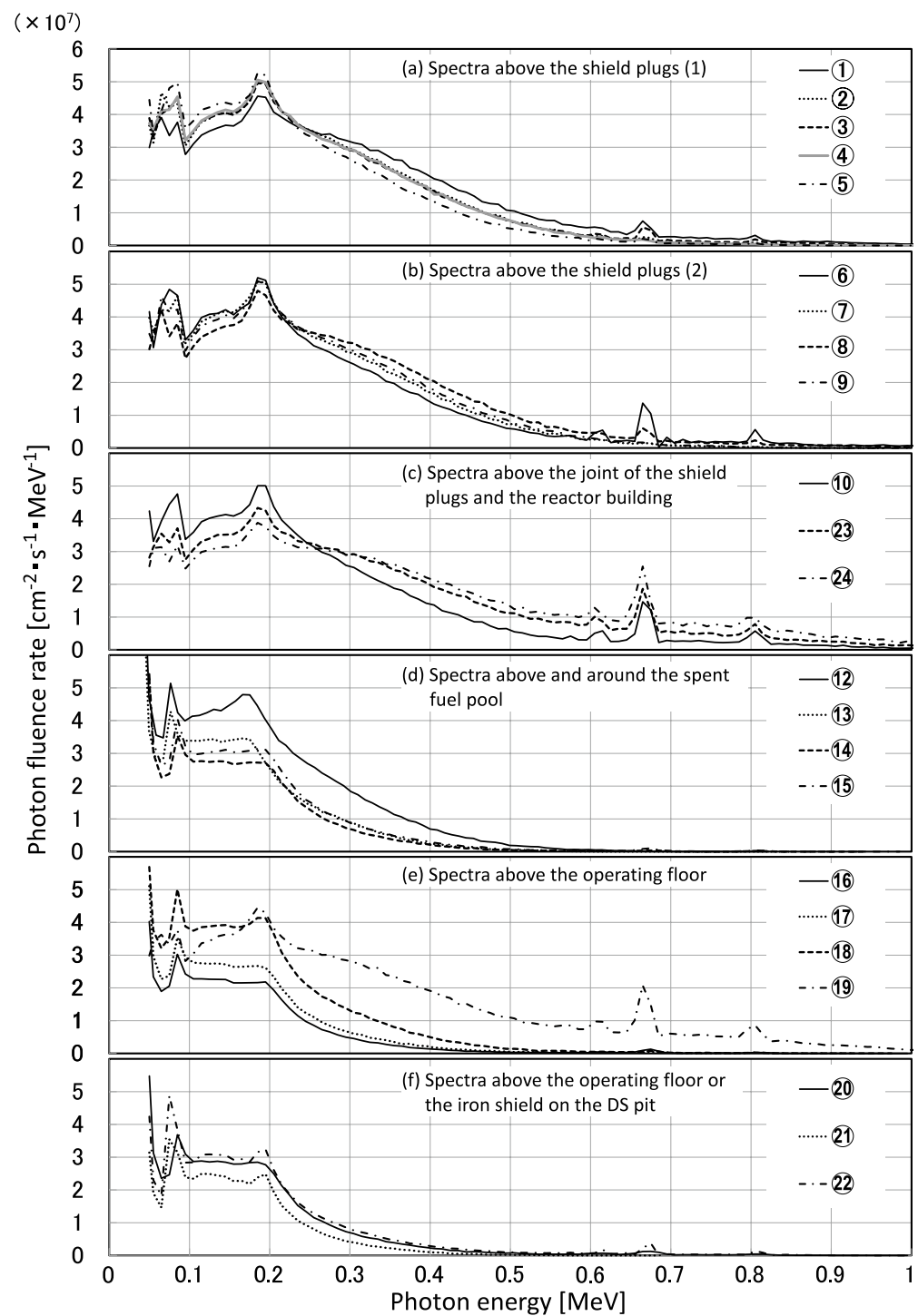

Figure 4 Photon energy spectra derived by unfolding the pulse height spectra measured above the operating floor of unit 3 reactor

Table 1 Relationship between the angle and the energy of Compton scattered photons

\begin{tabular}{cccccccc}
\hline $\begin{array}{c}\text { Scattering angle } \\
{[\text { degrees }]}\end{array}$ & 90 & 105 & 120 & 135 & 150 & 165 & 180 \\
\hline $\begin{array}{c}\text { Nuclide } \\
(\text { Energy })\end{array}$ & \multicolumn{7}{c}{ Scattered photon energy $[\mathrm{MeV}]$} \\
\hline $\begin{array}{c}{ }^{137} \mathrm{Cs} \\
(0.662 \mathrm{MeV}) \\
\begin{array}{c}{ }^{134} \mathrm{Cs} \\
(0.605 \mathrm{MeV}) \\
{ }^{134} \mathrm{Cs}\end{array} \\
(0.8 \mathrm{MeV})\end{array}$ & 0.288 & 0.252 & 0.225 & 0.206 & 0.194 & 0.187 & 0.184 \\
\hline
\end{tabular}


rays corresponds to the peak at approximately $0.2 \mathrm{MeV}$ observed in Figure 4, it would appear that the peak observed at approximately $0.2 \mathrm{MeV}$ is because of the photon beams scattered in the air above the collimator.

\section{Summary}

We measured the photon energy spectra on the operating floor of the Unit 3 reactor at Fukushima Daiichi Nuclear Power Station using a CdZnTe semiconductor detector and lead collimator. No photoelectric peak of the $\gamma$-rays from any radioactive nuclides, other than ${ }^{137} \mathrm{Cs}$ and ${ }^{134} \mathrm{Cs}$, was observed and it was estimated that radioactive Cs was the main source. In addition, from the analysis of the photon energy spectra derived from the response analysis of MCNP-4C code and the unfolding process with MAXED code, it was confirmed that the low-energy scattered component made a large contribution and the influence of direct radiation was low. The photon beam energy spectra obtained could be the basic data required for evaluating the dose reduction effect of a shield plate placed on the operating floor. In future studies, we will evaluate scattered rays in detail to estimate the location of the radiation source that contributes the most to the radiation dose based on the evaluation results.

We would like to express our deep gratitude for the advice and cooperation of the officials of the Secretariat of the Nuclear Regulation Authority, High Energy Accelerator Research Organization, Tokyo Electric Power Company, Toshiba and Kashima JV in this study.

\section{References}

1) Tokyo Electric Power Company, Fukushima Nuclear Accident Analysis Report, Tokyo Electric Power Company (20 Jun 2012). http://www.tepco.co.jp/en/press/corp-com/release/betu12_e/images/120620e0104.pdf

2) Tokyo Electric Power Company, Removal of nuclear fuel from spent fuel pool of unit 3 reactor at Fukushima Daiichi Nuclear Power Station, Handout for the 36th Commission on Supervision and Evaluation of the Specified Nuclear Facility (1 Jul 2015). [in Japanese]. http://www.nsr.go.jp/data/000112688.pdf

3) Kromek Group PLC, GR1 Spec Sheet; Revision 10, Kromek Group PLC (2015). http://www.kromek. com/index.php/products/nuclear-technology/czt/gr1-gamma-ray-spectrometer

4) J. F. Briesmeister (ed.), MCNP-A General Monte Carlo N-Particle Transport Code, Version 4C, LA13709-M, Los Alamos National Laboratory (2000).

5) M. Reginatto, P. Goldhagen, S. Neumann, "Spectrum unfolding, sensitivity analysis and propagation of uncertainties with the maximum entropy deconvolution code MAXED," Nucl. Instr Meth. A476, 242-246 (2002).

6) Secretariat of Nuclear Regulation Authority, Investigation of Radiation Sources on Operating Floor of unit 3 Reactor at Fukushima Daiichi Nuclear Power Station, Handout for the 38th Commission on Supervision and Evaluation of the Specified Nuclear Facility (18 Dec 2015). [in Japanese]. http://www. nsr.go.jp/data/000133830.pdf 